\title{
An ROI/xROI Based Rate Control Algorithm in H.264|AVC for Video Telephony Applications
}

\author{
Changhee $\mathrm{Kim}^{1}$, Taeyoung $\mathrm{Na}^{1}$, Jeongyeon $\mathrm{Lim}^{2}$, Youngho $\mathrm{Joo}^{2}$, Kimun $\mathrm{Kim}^{2}$, \\ Jaewoan Byun ${ }^{2}$, and Munchurl Kim ${ }^{1}$ \\ ${ }^{1}$ School of Engineering, Information and Communications University \\ 119 Munjiro, Yuseong-gu, Daejeon, 305-732, Korea \\ \{changhee21c, tyna, mkim\} @icu.ac.kr \\ ${ }^{2}$ Core Network Development Team, Institute of Network Technology, SK Telecom \\ Soonae-dong, Bundang-gu, Sungnam, Kyunggido, 463-784, Korea \\ \{jylim, yhzoo, kmkim, jbyun\} @sktelecom.com
}

\begin{abstract}
Channel bandwidth in $3 \mathrm{G}$ communication networks is very much limited for video telephony services. Therefore, it is worthwhile to enhance a subjective quality of video contents via ROI based coding. In this paper, An $\mathrm{ROI} / \mathrm{xROI}$ based rate-control method is proposed, which considers the coding of both the ROI and the extended ROIs (xROI's) in non-ROI to meet given target bitrates. In the proposed method, the $\mathrm{QP}$ values are increasingly assigned in MB wide inside the non-ROI away from ROI. This reduces the abrupt change in visual quality and the amounts of residual signals along the border between ROI and non-ROI. In this regard, the subjective visual quality is enhanced as well as the proposed rate control has flexibility to control the amounts of the output bitstreams. Experimental results show that the proposed scheme can more effectively achieve the average target bitrates with the better subjective quality than the existing rate control algorithm in H.264IAVC by reducing the variation of the output bitstream amounts.
\end{abstract}

Keywords: ROI, xROI, rate control, H.264IAVC, video telephony.

\section{Introduction}

The H.264IAVC standard has been developed by the Joint Video Team (JVT), which was jointly established by ISO/IEC MPEG and ITU-T VCEG [1]. The H.264IAVC enhances coding efficiency by adopting some innovative features such as $4 \times 4$ integer transform, multiple reference frames, various block types for $\mathrm{MC}$, deblocking filters, and a rate-distortion optimization (RDO) based mode decision [2].

The Baseline profile of H.264IAVC is largely intended for mobile video telephony and mobile TV applications which require real-time transmission via limited channel bandwidths. Constant bit-rate channels can not deal with variable bitrates data stream of H.264IAVC in real-time unless an appropriate rate control mechanism is incorporated. Rate control algorithms adaptively adjust quantization parameter $(\mathrm{QP})$ values to accomplish required target bitrates [3]. 
H.264IAVC utilizes RDO to select an optimal mode for each MB, which causes the rate control to be more complicated. In H.264|AVC, the RDO based coding requires $\mathrm{QP}$ assignment a priori, and QP is determined by using Mean Absolute Differences (MAD). However, the MAD of the current MB is available only after RDO based coding is completed. This problem is called 'Chicken and Egg Dilemma'. In order to solve this dilemma, the rate control algorithm for H.264IAVC uses a linear model to estimate MAD of each MB in the current frame from that of co-located MB in the previous frame, and adopts a quadratic Rate-Distortion (R-D) model to predict QP. This predicted QP may not be accurate because the estimated information is not completely associated with the current frame [4]. Therefore, the fluctuation of output bitrates is high, and QP dependency of RDO causes the rate control algorithm of H.264IAVC to be computationally expensive. To solve these problems, a lot of studies have been accomplished by using other models in [5]-[7].

Recently, the demands for video telephony services on mobile devices are increasing in the 3rd Generation $(3 \mathrm{G})$ communication networks. The channel bandwidth for each user is $64 \mathrm{kbps}$ at maximum. For video transmission, approximately $48 \mathrm{kbps}$ is used at most in circuit-switched channels. H.263 is currently adopted for video telephony services, but the video quality is not good enough to satisfy user's needs. To overcome this situation, substituting H.263 with H.264IAVC has been tried, yet it is difficult to directly employ the existing H.264IAVC. Especially, the current rate control algorithm for H.264|AVC should be improved because of the large fluctuation of bitrates and high complexity. Furthermore, Region-Of-Interest (ROI) based coding is a good means to maintain the visual quality of important parts in video at somewhat satisfactory levels under such a low bit-rate environment [8]. In [9]-[10], ROI based rate control algorithms have been proposed. However, these papers focused on the improvement of subjective visual quality by utilizing the ROI based coding which divides a frame only into two regions: ROI and non-ROI.

In this paper, an effective ROI/XROI based rate control algorithm compatible with H.264IAVC is introduced for video telephony applications. The xROI stands for extended ROI and non-ROI is divided into multiple xROI's in MB wide around ROI. The proposed scheme incorporates a simple update rule for assigning QP values to reduce the fluctuation of output bitrates in GOP. The QP values to be applied for the xROI's inside the non-ROI are increasingly assigned away from ROI so that the visual quality is smoothly degraded. The rest of this paper is organized as follows: In Section 2, the ROI/xROI based coding is introduced. The proposed rate control algorithm is addressed in Section 3, and experimental results are given in Section 4. Finally, we conclude this paper in Section 5.

\section{ROI/xROI Based Coding}

The existing ROI based coding splits a frame into two regions including ROI and non-ROI [8]. On the other hand, a large difference in visual quality between ROI and non-ROI can make the reconstructed frames unpleasant. To solve this drawback, the $\mathrm{QP}$ values are increasingly assigned for the xROI's (in MB-wide strip regions) in nonROI away from ROI. This also reduces the amounts of residual signals along the border between ROI and non-ROI so that coding efficiency is increased. 
We improve subjective visual quality using the ROI/xROI based coding. When xROI is applied into non-ROI, non-ROI is split into more than two areas. Fig. 1 shows an example of partitioning a frame into ROI (marked as (1)) and non-ROI which is further divided into multiple xROI's, that is, $\mathrm{xROI}_{1}, \mathrm{xROI}_{2}$ and $\mathrm{xROI}_{3}$, marked as (2), (3) and (4), respectively.
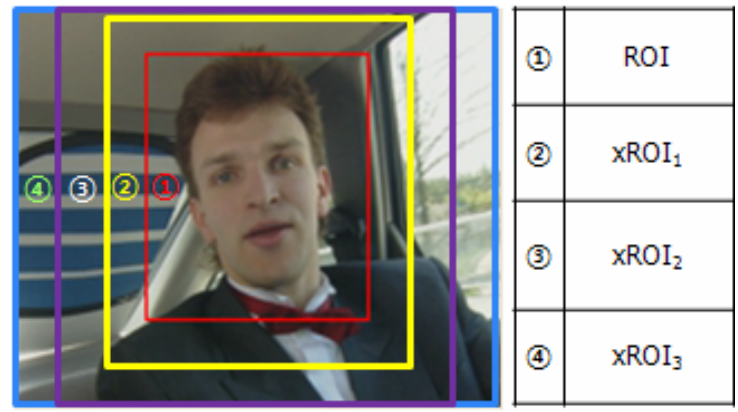

Fig. 1. Example of a frame in which the ROI/xROI based coding is employed for Car phone sequence / QCIF, ROI Size: 30MBs

In this scheme, the lowest $\mathrm{QP}$ value is assigned to $\mathrm{ROI}\left(\mathrm{QP}_{1}\right)$ and $\mathrm{QP}$ is increasingly assigned to the regions, $x \mathrm{xOI}_{1}\left(\mathrm{QP}_{2}\right), \mathrm{xROI}_{2}\left(\mathrm{QP}_{3}\right)$ and $\mathrm{xROI} \mathrm{I}_{3}\left(\mathrm{QP}_{4}\right)$, respectively.

$$
Q P_{1} \leq Q P_{2} \leq Q P_{3} \leq Q P_{4}
$$

So, the visual quality of the reconstructed frame is gracefully degraded away from the ROI. Then, QP of each XROI is increased by a constant value (QP-Step) depending on the distance from the ROI. So, ROI has the best quality and the area closer to ROI gets the better quality. This can enhance both the subjective and the objective video quality in comparison with the existing ROI based coding.

\section{Proposed Rate Control Algorithm}

\subsection{Overall Algorithm}

The proposed ROI/xROI based rate control algorithm performs a frame-level adjustment on the QP values (QP values for ROI and xROI's in non-ROI) to reduce the difference between the amounts of target bit and output bit and the bit fluctuation per a Group of Pictures (GOP) as well as the computational complexity, compared to the existing rate control algorithm in H.264IAVC. Fig. 2 illustrates the flow chart of the proposed ROI/XROI based rate control method.

Stage 1: Allocate a target bits for I- and P-pictures at frame-level.

Stage 2: Compute the remaining bit amount (=target bits-output bits of the previous frame) for the current frame and accumulate these remaining bits within the GOP. 


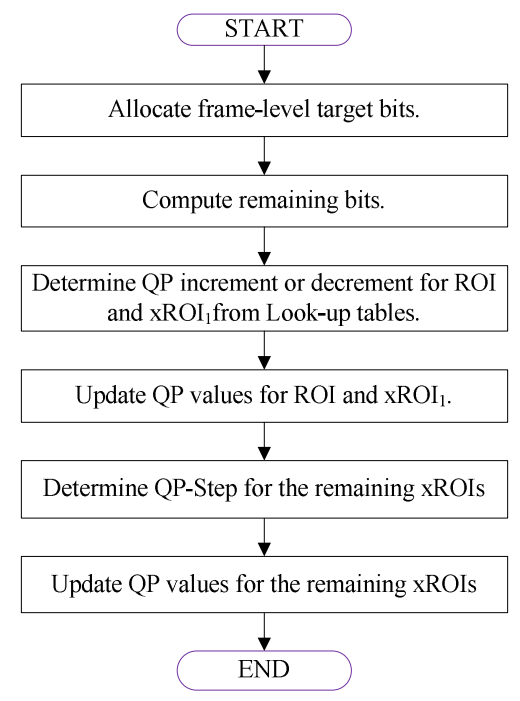

Fig. 2. Flow Chart of the Proposed ROI/xROI based Rate Control Algorithm

Stage 3: Determine QP increment or decrement for ROI and $\mathrm{xROI}_{1}$ from Lookup tables by taking into account the remaining bits of the previous frame and the accumulated remaining bits in the current GOP.

Stage 4: Update QP values for ROI and $\mathrm{xROI}_{1}$ using the QP increment or decrement in Stage 3.

Stage 5: Determine QP-Step for the remaining xROI's by taking into account the $\mathrm{QP}$ value for $\mathrm{xROI}_{1}$.

Stage 6: Update QP values for the remaining xROI's using QP-Step in Stage 5.

\subsection{Frame-Level Update of the QP Values for I- and P-Pictures}

As explained in the previous section, we need to adjust the QP values to be applied for coding ROI and xROI's in non-ROI to meet the target bit rates. Video telephony may require video transmission of QCIF size in average of $10 \mathrm{fps}$ via the circuitswitched channel of $42 \mathrm{kbps}$ for satisfactory services. In this case, we may have each GOP with 10 frames including one I-frame and 9 P-frames. Our goal is to meet the target bitrates per GOP with the output bitrates. The QP values are renewed on a frame-level to meet the average target bitrates. An encoding target bit amount is assigned for the I-picture in the first GOP. The excess or remnant bit amount for each Ipicture is computed for a given target bitrates (42kbps in our case) after I-picture encoding, which is also taken into account when the OP values for ROI and xROI's in the next I-picture are to be adjusted. The remaining bits after the I-picture encoding is equally divided and distributed for the following P-pictures as their target bitrates in the current GOP. For each P-picture, adjustment of QP values for ROI and xROI's is done depending on the excess or remnant bit for the P-picture encoding as well as the accumulated amount of the excess and remnant bits until the previous P-pictures in GOP. The adjustment of QP increments and decrements is made by a look-up table 
based on the amounts of the remaining bit amount in the previous P-picture and the accumulated bit amount until the previous in each GOP, which is experimentally determined by various training sequences with slow and fast motion.

We assume that the ROI is already selected. The ROI includes a face, consisting of $30 \mathrm{MBs}$. For this, we set an appropriate ROI in the first frame of each sequence and fix its position during encoding. Automatic segmentation of ROI is outside the scope of this paper. A detailed explanation of the ROI/xROI based rate control algorithm is given as follows:

\section{Stage 1: Frame-level Target Bits Allocation}

If (The current frame $==$ I-frame) \{

The target bit amount for an I-frame in a GOP is computed as

$$
R_{G O P, t \arg e t}=R_{t \arg e t}, R_{I, t \arg e t}=\alpha_{I} \cdot R_{G O P, t \arg e t}
$$

where $R_{G O P, t \arg e t}, R_{t \arg e t}$ are the target bitrates for a GOP and a sequence, respectively. $\alpha_{I}$ is selected experimentally from various video sequences with $\alpha_{I}=0.35$.

\}

Else\{

If (The previous frame $==$ I-frame)

The amount of the target bits for P-frame in $i$-th GOP is derived as

$$
R_{P, t \arg e t}^{i}=\left(R_{G O P, t \arg e t}-R_{I, \text { output }}^{i}\right) / N_{P, \text { frame }}^{G O P}
$$

where $R_{I, \text { output }}^{i}$ is the number of the output bits of I-frame in $i$-th GOP, $N_{P \text {, frame }}^{G O P}$ is the number of P-frames in a GOP and is set to 9 in our case.

\}

\section{Stage 2: Computation of Remaining Bits}

If (The current frame $==$ I-frame)

The remaining bits of I-frame in $i$-th GOP, $G_{I, \text { frame }}^{i}$ are calculated as

$$
G_{I, \text { frame }}^{i}=R_{I, t \arg e t}-R_{I, \text { output }}^{i-1}
$$

Else \{

If (The current frame $==$ The first $\mathrm{P}$-frame in sequence)

$$
G_{P, G O P}^{1,1}=0
$$

where $G_{P, G O P}^{1,1}$ is the initial accumulated remaining bits for the first P-frame in the $1^{\text {st }} \mathrm{GOP}$.

Else if (The previous frame $==$ I-frame)

$$
G_{P, G O P}^{i, 1}=G_{P, G O P}^{i-1, N_{P, \text { frame }}^{G O P}}
$$

Else\{ 
The remaining bits of ( $n$-1)-th P-frame in $i$-th GOP are provided by

$$
G_{P, \text { frame }}^{i, n-1}=R_{P, t \arg e t}^{i}-R_{P, \text { output }}^{i, n-1}(n>=2)
$$

where $R_{P, \text { output }}^{i, n-1}$ is the amount of the output bits after encoding the (n-1)-th Pframe in $i$-th GOP. Then, we accumulate the remaining bits from the first Pframe to (n-1)-th P-frame in $i$-th GOP. The accumulated remaining bits for $n$-th $\mathrm{P}$-frame in $i$-th GOP are given by

$$
G_{P, G O P}^{i, n}=\sum_{k=1}^{n-1} G_{P, \text { frame }}^{i, k}(n>=2)
$$

\}

$$
\text { \} }
$$

\section{Stage 3: Determination of QP increment or decrement amount from Look-up tables}

If (The current frame $==\mathrm{P}$-frame $\& \&$ The Previous frame != I-frame $)\{$

The amounts of QP increment/decrement for ROI and $\mathrm{xROI}_{1}$ are decided as follows:

$$
\begin{aligned}
\text { If }\left(P S N R_{P, \mathrm{ROI}}^{i, n-1}<P S N R_{P, \mathrm{xROI}}^{i, n-1} \& \& P S N R_{P, \mathrm{ROI}}^{i, n-1}>P S N R_{-} T h_{1}\right) \\
\Delta Q P_{p, \mathrm{ROI}}^{i, n}=Q p_{\mathrm{ROI}}^{1}, \Delta Q P_{p, \mathrm{xROI}_{1}}^{i, n}=Q p_{\mathrm{xROI}_{1}}^{1}
\end{aligned}
$$

where $P S N R_{P, \mathrm{ROI}}^{i, n-1}$ and $P S N R_{P, \mathrm{xROI}}^{i, n-1}$ are the PSNR values of (n-1)-th P-frame in $i$-th GOP for $\mathrm{ROI}$ and $\mathrm{xROI}_{1}$, respectively. Accordingly, $\Delta Q P_{p, \mathrm{ROI}}^{i, n}$ and $\Delta Q P_{p, \mathrm{xROI}_{1}}^{i, n}$ are the QP changes for $n$-th P-frame in $i$-th GOP for ROI and $\mathrm{xROI}_{1}$, respectively. $Q p_{\mathrm{ROI}}^{1}$ and $Q p_{\mathrm{xROI}}^{1}$ are the $\mathrm{QP}$ changes defined in Table 1 for ROI and $\mathrm{XROI}_{1}$, respectively. $P S N R \_T h_{1}$ is a PSNR threshold for ROI. PSNR_Th 1 is set to $35 \mathrm{~dB}$ in our case.

Else if $\left(P S N R_{P, \mathrm{ROI}}^{i, n-1}<P S N R \_T h_{2} \& \& G_{P, G O P}^{i, n}>\beta_{1}\right)$

$$
\Delta Q P_{p, \mathrm{ROI}}^{i, n}=Q p_{\mathrm{ROI}}^{2}, \Delta Q P_{p, \mathrm{xROI}_{1}}^{i, n}=Q p_{\mathrm{xROI}_{1}}^{2}
$$

where $P S N R \_T h_{2}$ is a PSNR threshold for ROI. PSNR_Th is set to $33 \mathrm{~dB}$ in our case. $\beta_{1}$ is a threshold for the accumulated remaining bits in GOP and is determined by experiments with $\beta_{1}=-500 . Q p_{\mathrm{ROI}}^{2}$ and $Q p_{\mathrm{xROI}_{1}}^{2}$ are the $\mathrm{QP}$ changes defined in Table 1 for ROI and $\mathrm{xROI}_{1}$, respectively.

Else

If $\left(G_{P, G O P}^{i, n}<0\right)\left\{\Delta Q P_{p, \mathrm{ROI}}^{i, n}=Q p_{\mathrm{ROI}}^{3}, \Delta Q P_{p, \mathrm{xROI}_{1}}^{i, n}=Q p_{\mathrm{xROI}_{1}}^{3}\right.$, Go to Step 1$\}$

Else $\quad\left\{\Delta Q P_{p, \mathrm{ROI}}^{i, n}=Q p_{\mathrm{ROI}}^{4}, \Delta Q P_{p, \mathrm{xROI}_{1}}^{i, n}=Q p_{\mathrm{xROI}_{1}}^{4}\right.$, Go to Step 2$\}$

where $Q p_{\mathrm{ROI}}^{3}, Q p_{\mathrm{ROI}}^{4}$ and $Q p_{\mathrm{xROI}_{1}}^{3}, Q p_{\mathrm{xROI}}^{4}$ are the $\mathrm{QP}$ changes defined in Table 1 \} for ROI and $\mathrm{xROI}_{1}$, respectively. 
Table 1 gives the values of $Q p_{\mathrm{ROI}}, Q p_{\mathrm{xROI}}$ depending on $G_{P, G O P}^{i, n}, G_{P, \text { frame }}^{i, n-1} \cdot Q P_{\mathrm{x}}$ is the

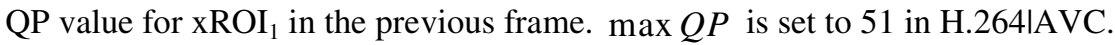

Table 1. QP Adjustment Table for P-frames

$$
\left(G_{G O P}=G_{P, G O P}^{i, n}, G_{\text {frame }}=G_{P, \text { frame }}^{i, n-1}, Q P_{\mathrm{x}}=Q P_{P, \mathrm{xROI}_{1}}^{i, n-1}\right)
$$

\begin{tabular}{|c|c|c|c|c|c|}
\hline Condition & $Q p_{\mathrm{ROI}}^{1}$ & $Q p_{\mathrm{xROI}_{1}}^{1}$ & Condition & $Q p_{\mathrm{ROI}}^{4}$ & $Q p_{\mathrm{xROI}_{1}}^{4}$ \\
\hline · & -5 & +10 & if $\left(G_{G O P}>3000\right)$ & -2 & -5 \\
\hline Condition & $Q p_{\mathrm{ROI}}^{2}$ & $Q p_{\mathrm{xROI}_{1}}^{2}$ & else_if $\left(G_{G O P}>2000\right)$ & -1 & -3 \\
\hline if $\left(G_{G O P}>5000\right)$ & -5 & -7 & else_if $\left(G_{G O P}>1000\right)$ & 0 & -3 \\
\hline else_if $\left(G_{G O P}>3000\right)$ & -4 & -5 & Condition & $Q p_{\mathrm{ROI}}^{4 a}$ & $Q p_{\mathrm{xROI}_{1}}^{4 a}$ \\
\hline else_if $\left(G_{G O P}>1000\right)$ & -3 & -4 & $i f\left(G_{G O P}>3000\right)$ & -3 & 0 \\
\hline else & -3 & +5 & else_if $\left(G_{G O P}>2000\right)$ & -1 & -2 \\
\hline Condition & $Q p_{\mathrm{ROI}}^{3}$ & $Q p_{\mathrm{xROI}_{1}}^{3}$ & else_if $\left(G_{G O P}>1000\right)$ & -1 & -2 \\
\hline if $\left(G_{G O P}<-2000\right)$ & +4 & +6 & Condition & $Q p_{\mathrm{ROI}}^{4 b}$ & $Q p_{\mathrm{xROI}_{1}}^{4 b}$ \\
\hline else_if $\left(G_{G O P}<-500\right)$ & +2 & +4 & if $\left(G_{G O P}>4000\right)$ & -3 & -3 \\
\hline else_if $\left(G_{G O P}<-100\right)$ & +1 & +3 & else_if $\left(G_{G O P}>3000\right)$ & -2 & -1 \\
\hline else & 0 & +2 & else_if $\left(G_{G O P}>2000\right)$ & -1 & +1 \\
\hline Condition & $Q p_{\mathrm{ROI}}^{3 a}$ & $Q p_{\mathrm{xROI}_{1}}^{3 a}$ & else_if $\left(G_{G O P}>1500\right)$ & -2 & +1 \\
\hline if $\left(G_{G O P}<-2000\right)$ & +1 & $\max Q P-Q P_{\mathrm{x}}$ & else_if $\left(G_{G O P}>1000\right)$ & -1 & +3 \\
\hline else_if $\left(G_{G O P}<-1000\right)$ & +2 & $\max Q P-Q P_{\mathrm{x}}$ & Else & 0 & -1 \\
\hline else_if $\left(G_{G O P}<-500\right)$ & +1 & +6 & Condition & $Q p_{\mathrm{ROI}}^{4 c}$ & $Q p_{\mathrm{xROI}_{1}}^{4 c}$ \\
\hline else_if $\left(G_{G O P}<-300\right)$ & +1 & +2 & if $\left(G_{G O P}>3000\right)$ & -1 & -2 \\
\hline else & +1 & +1 & else_if $\left(G_{G O P}>2000\right)$ & -1 & -2 \\
\hline Condition & $Q p_{\mathrm{ROI}}^{3 b}$ & $Q p_{\mathrm{xROI}}^{3 b}$ & $\begin{array}{l}\text { else_if }\left(G_{G O P}>1000\right. \\
\left.\& \& G_{\text {frame }}>0\right)\end{array}$ & -1 & -2 \\
\hline$\cdot$ & +1 & $\max Q P-Q P_{\mathrm{x}}$ & else_if $\left(G_{G O P}>1000\right)$ & 0 & -2 \\
\hline & & & $\begin{array}{l}\text { else } e_{-} \text {if } \\
\left(G_{\text {frame }}<-2000\right)\end{array}$ & +1 & +2 \\
\hline
\end{tabular}

Step 1: Fine-tuning of QP changes when the accumulated remaining bits in GOP are negative

$$
\text { If }\left(n==N_{P, \text { frame }}^{G O P} \& \& G_{P, \text { frame }}^{i, n-1}<\beta_{2}\right) \Delta Q P_{p, \mathrm{ROI}}^{i, n}+=Q p_{\mathrm{ROI}}^{3 a}, \Delta Q P_{p, \mathrm{xROI}_{1}}^{i, n}+=Q p_{\mathrm{xROI}_{1}}^{3 a}
$$

where $n$ is the index of the current frame, and $\beta_{2}$ is a threshold for the remaining bits of the previous P-frame in $i$-th GOP and is experimentally determined with $\beta_{2}=1500 . Q p_{\mathrm{ROI}}^{3 a}$ and $Q p_{\mathrm{xROI}_{1}}^{3 a}$ are the $\mathrm{QP}$ changes defined in Table 1 for ROI and $\mathrm{xROI}_{1}$, respectively. 
Else\{ If $\left(n==N_{P, \text { frame }}^{G O P}-1 \& \& G_{P, G O P}^{i, n}<\beta_{3}\right)$

$$
\left.\Delta Q P_{p, \mathrm{ROI}}^{i, n}+=Q p_{\mathrm{ROI}}^{3 b}, \Delta Q P_{p, \mathrm{xROI}_{1}}^{i, n}+=Q p_{\mathrm{xROI}_{1}}^{3 b}\right\}
$$

where $\beta_{3}$ is a threshold for the accumulated remaining bits for the current frame in $i$-th GOP and is experimentally determined with $\beta_{3}=-2000 . Q p_{\mathrm{ROI}}^{3 b}$ and $Q p_{\mathrm{xROI}}^{3 b}$ are the $\mathrm{QP}$ changes defined in Table 1 for $\mathrm{ROI}$ and $\mathrm{xROI}_{1}$, respectively.

Step 2: Fine-tuning of QP changes when the accumulated remaining bits in GOP are positive

$$
\begin{aligned}
& \text { If }\left(G_{P, G O P}^{i, n}>\beta_{4} \& \& G_{P, \text { frame }}^{i, n-1}>\beta_{5}\right) \\
& \text { If }\left(n==N_{P, \text { frame }}^{G O P}\right) \quad \Delta Q P_{p, \mathrm{ROI}}^{i, n}+=Q p_{\mathrm{ROI}}^{4 a}, \Delta Q P_{p, \mathrm{xROI}_{1}}^{i, n}+=Q p_{\mathrm{xROI}_{1}}^{4 a} \\
& \text { Else } \quad \Delta Q P_{p, \mathrm{ROI}}^{i, n}+=Q p_{\mathrm{ROI}}^{4 b}, \Delta Q P_{p, \mathrm{xROI}_{1}}^{i, n}+=Q p_{\mathrm{xROI}_{1}}^{4 b}
\end{aligned}
$$

where $\beta_{4}$ and $\beta_{5}$ are the threshold values for the accumulated remaining bits until the previous frame, and for the remaining bits of the previous frame after encoding in $i$-th GOP, respectively. They are experimentally determined with $\beta_{4}=4,000$ and $\beta_{5}=300 . Q p_{\mathrm{ROI}}^{4 a}, Q p_{\mathrm{ROI}}^{4 b}$ and $Q p_{\mathrm{xROI}_{1}}^{4 a}, Q p_{\mathrm{xROI}_{1}}^{4 b}$ are the $\mathrm{QP}$ changes defined in Table 1 for ROI and $\mathrm{xROI}_{1}$, respectively.

Else $\left\{\right.$ If $\left.\left(n==N_{P, \text { frame }}^{G O P}\right) \Delta Q P_{p, \mathrm{ROI}}^{i, n}+=Q p_{\mathrm{ROI}}^{4 c}, \Delta Q P_{p, \mathrm{xROI}_{1}}^{i, n}+=Q p_{\mathrm{xROI}_{1}}^{4 c}\right\}$

\section{Stage 4: Update of QP values for ROI and $x \mathrm{ROI}_{1}$}

If (The current frame $==I$-frame $)\{$

If (The current frame $==$ The first I-frame in the sequence)

$$
Q P_{I, \mathrm{ROI}}^{1}=Q P_{\mathrm{ROI}}^{\text {init }}, Q P_{I, \mathrm{xROI}{ }_{1}}^{1}=Q P_{\mathrm{xROI}_{1}}^{\text {init }}
$$

where $Q P_{\mathrm{ROI}}^{\text {init }}$ and $Q P_{\mathrm{xROI}_{1}}^{\text {init }}$ are the initial $\mathrm{QP}$ values for $\mathrm{ROI}$ and $\mathrm{xROI}_{1}$ in the first I-frame of the sequence. $Q P_{\mathrm{ROI}}^{\text {init }}$ and $Q P_{\mathrm{xROI}}^{\text {init }}{ }_{1}$ are set to 30 and 35, respectively, in our experiments.

Else \{

$$
\begin{aligned}
& \text { If }\left(\left|G_{I, \text { frame }}^{i-1}\right|>\alpha_{G} \cdot R_{I, \text { target }}\right) \\
& \quad Q P_{I, \mathrm{ROI}}^{i}=\left(\sum_{n=1}^{N_{P, \text { frame }}^{G O P}} Q P_{P, \mathrm{ROI}}^{i-1, n}\right) / N_{P, \text { frame }}^{G O P}, Q P_{I, \mathrm{XROI}}^{i}=\left(\sum_{n=1}^{N_{P, \text { frame }}^{G O P}} Q P_{P, \mathrm{RRO} \mathrm{I}_{1}}^{i-1, n}\right) / N_{P, \text { frame }}^{G O P}
\end{aligned}
$$

Else $\quad Q P_{I, \mathrm{ROI}}^{i}=Q P_{I, \mathrm{ROI}}^{i-1}, Q P_{I, \mathrm{xROI}_{1}}^{i}=Q P_{I, \mathrm{xROI}_{1}}^{i-1}(i>=2)$

where $\alpha_{G}$ is a threshold determined by experiments with $\alpha_{G}=0.4 . Q P_{P, \text { ROI }}^{i-1, n}$, $Q P_{P, x \mathrm{ROI}}^{i-1, n}$ are the QP values for ROI and $\mathrm{xROI}_{1}$ of $n$-th P-frame in $(i-1)$-th GOP. 


\section{Else \{}

If (The previous frame $==\mathrm{I}$-frame)

$$
Q P_{P, \mathrm{ROI}}^{i, 1}=Q P_{I, \mathrm{ROI}}^{i}, Q P_{P, \mathrm{xROI}}^{i, 1}=Q P_{I, \mathrm{xROI}_{1}}^{i}
$$

Else

$$
\begin{aligned}
& Q P_{P, \mathrm{ROI}}^{i, n}=Q P_{P, \mathrm{ROI}}^{i, n-1}+\Delta Q P_{p, \mathrm{ROI}}^{i, n} \\
& Q P_{P, \mathrm{xROI}_{1}}^{i, n}=Q P_{P, \mathrm{xROI}_{1}}^{i, n-1}+\Delta Q P_{p, \mathrm{xROI}_{1}}^{i, n}
\end{aligned}
$$

\section{Stage 5: Determination QP-Step for the remaining $x$ ROI's}

The QP-Step of $n$-th P-frame in i-th GOP is computed by

$$
S_{\mathrm{xROI}}^{i, n}=\left(\max \mathrm{QP}-Q P_{\mathrm{xROI}}^{i, n}\right) /\left(N_{\text {region }}^{\mathrm{xROI}}-1\right)
$$

where $N_{\text {region }}^{\mathrm{xROI}}$ is the number of divided xROI regions in a frame. The QP-Step is bounded by the maximum value set to 5 .

\section{Stage 6: Update of $Q P$ values for the remaining $x R O I$ 's}

$\operatorname{For}\left(m=2 ; m<N_{\text {region }}^{\text {xROI }} ; m++\right)$

$$
Q P_{\mathrm{xROI}_{m}}^{i, n}=Q P_{\mathrm{xROI}_{1}}^{i, n}+S_{\mathrm{xROI}}^{i, n} \cdot(m-1)
$$

\section{Experimental Results}

We implement the proposed ROI/xROI based rate control algorithm in the reference software, JM 11.0 [12]. The experiments are performed based on the Baseline profile of H.264/AVC with RDO ON, search range \pm 16 , intra period 10, the Hadamard transform ON, and FMO type 2. The five sequences were used in the experiments, including Carphone $(C)$, Foreman $(F)$, Silent $(S)$, Salesman $(M)$, and Grandma $(G)$ with

\begin{tabular}{|c|c|c|c|c|c|c|c|c|c|}
\hline \multirow{2}{*}{ Seq. } & \multirow{2}{*}{ values } & \multicolumn{4}{|c|}{ Original JM 11.0} & \multicolumn{4}{|c|}{ Proposed method } \\
\hline & & ROI & $\mathrm{xROI}_{1}$ & $\mathrm{xROI}_{2}$ & $\mathrm{xROI}_{3}$ & ROI & $\mathrm{xROI}_{1}$ & $\mathrm{xROI}_{2}$ & $\mathrm{xROI}_{3}$ \\
\hline \multirow{2}{*}{$C$} & Y-PSNR & 32.54 & 34.05 & 34.61 & 33.3 & 34.81 & 31.80 & 30.17 & 26.66 \\
\hline & bitrates & \multicolumn{4}{|c|}{45.78} & \multicolumn{4}{|c|}{41.51} \\
\hline \multirow{2}{*}{$F$} & Y-PSNR & 30.43 & 30.62 & 31.22 & 30.41 & 33.74 & 28.67 & 27.59 & 25.18 \\
\hline & bitrates & \multicolumn{4}{|c|}{42.1} & \multicolumn{4}{|c|}{41.34} \\
\hline \multirow{2}{*}{$S$} & Y-PSNR & 32.04 & 33.18 & 33.33 & 33.80 & 34.48 & 31.05 & 29.24 & 26.32 \\
\hline & bitrates & \multicolumn{4}{|c|}{42.06} & \multicolumn{4}{|c|}{41.24} \\
\hline \multirow{2}{*}{$M$} & Y-PSNR & 32.41 & 34.72 & 35.39 & 34.16 & 34.156 & 31.86 & 29.89 & 26.14 \\
\hline & bitrates & \multicolumn{4}{|c|}{42.57} & \multicolumn{4}{|c|}{41.59} \\
\hline \multirow{2}{*}{$G$} & Y-PSNR & 34.75 & 38.3 & 38.65 & 38.99 & 35.32 & 34.39 & 32.75 & 30.66 \\
\hline & bitrates & \multicolumn{4}{|c|}{43.19} & \multicolumn{4}{|c|}{41.96} \\
\hline
\end{tabular}
QCIF, 100 frames and 10fps. The PC for the simulation has Pentium4 3.0GHz CPU and 2.0Gbyte RAM.

Table 2. Comparison of the bitrates (kbps) and PSNR values (dB) in Each Region Between JM 11.0 and the Proposed Algorithm

(QCIF, 100frames, 10fps, GOP 10frames, Target Bitrates 42kbps, ROI Size: 30MBs). 
In order to evaluate the proposed algorithm, we compare the performance of our scheme with that of in the original JM 11.0. Table 2 gives the comparisons of the bitrates, and Y-PSNR values in each region between the original JM 11.0 and the proposed algorithm. In the proposed algorithm, it is shown that the video quality of $\mathrm{ROI}$ is the best and the region nearer to ROI obtains the better quality. This effect can elevate the overall perceptual quality. Since the bit amounts spent on ROI have been more increased for ROI with higher motion, the amounts of output bits for xROI's have been reduced accordingly to compensate them. Moreover, the Y-PSNR value of non-ROI (xROI's) is more sensitive than that of ROI because non-ROI usually has less motion than ROI. This is taken into account for QP adjustments in xROI's.
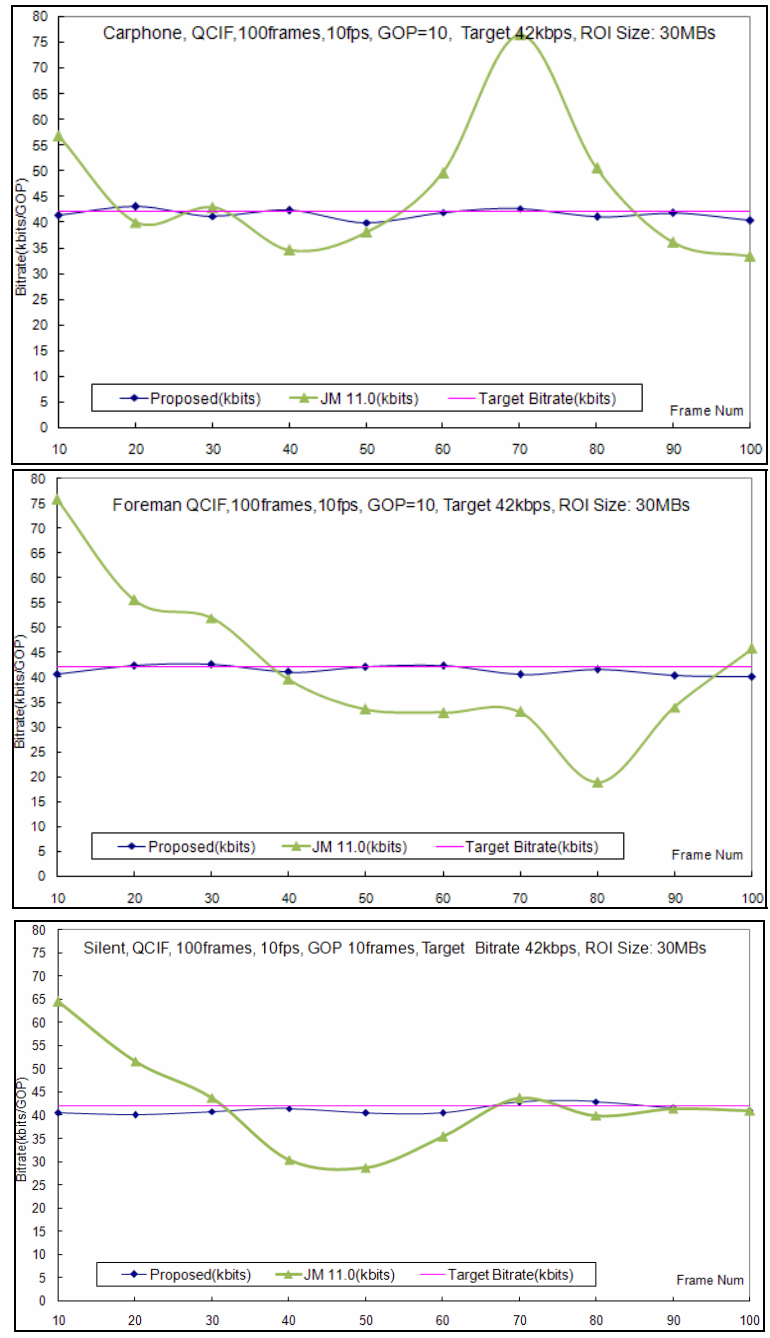

Fig. 3. Comparisons of the bitrates fluctuation per GOP between JM 11.0 and the proposed rate control algorithm for $C, F$ and $S$ sequences 
Fig. 3 shows the comparison of the bitrates per GOP on both methods for the sequences $(C, F$ and $S)$. As shown in Fig. 3 the proposed rate control algorithm yields output bitrates very close to the target bitrates per GOP and reduces the variation of output bitrates compared with the original rate control in JM 11.0.

Fig. 4 shows the comparison of the subjective quality between JM 11.0 and the proposed scheme. As shown in Fig. 4, it is demonstrated that the proposed algorithm outperforms the existing scheme of JM 11.0 in the perceived visual quality.
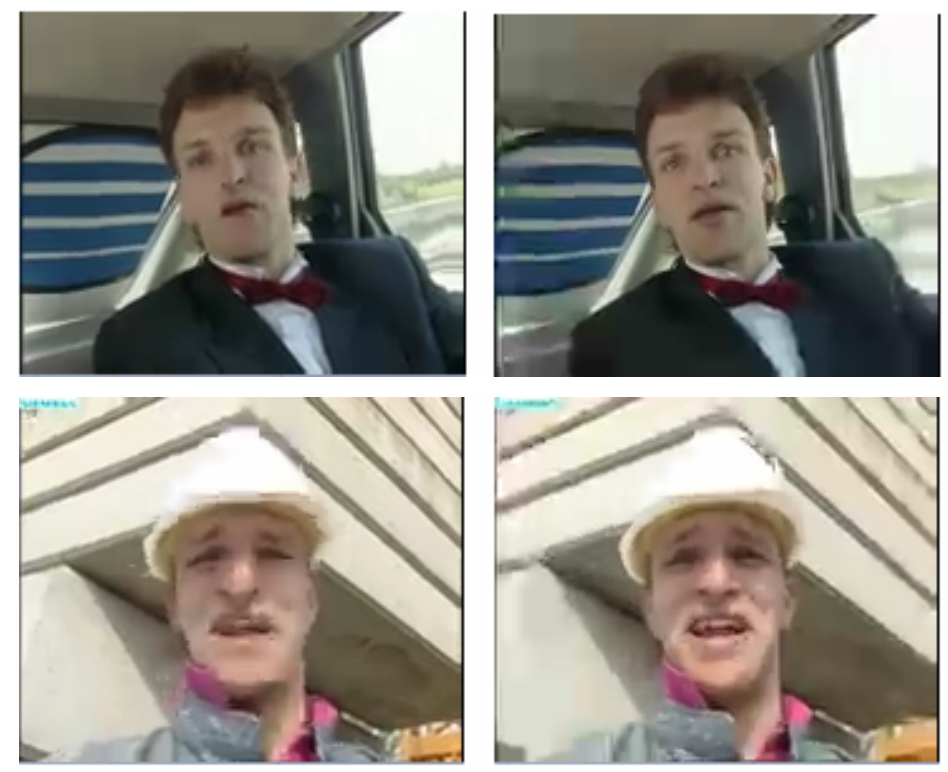

Fig. 4. Comparisons of the subjective quality between JM 11.0 (left) and the proposed rate control algorithm (right) in each sequence for $C$ (top) and $F$ (bottom) sequences

The additional experimental results are shown in Table 3. Table 3 tabulates the times taken for encoding, the standard deviations of PSNR values and the bitrates per GOP. The standard deviation implies the extent of the fluctuation. It is observed that the proposed algorithm can lower the temporal quality change with the significantly decreased fluctuation of output bitrates in comparison with the existing scheme in JM 11.0. Furthermore, the total encoding time can be saved up to approximately $5 \%$ of the encoding time in JM 11.0.

Table 3. Additional Results between JM 11.0 and the Proposed Algorithm

\begin{tabular}{|c|c|c|c|c|c|c|}
\hline Sequence & \multicolumn{2}{|c|}{ Std. of PSNR (dB) } & \multicolumn{2}{c|}{ Std. of Bitrates (kbits) } & \multicolumn{2}{c|}{ Encoding Times (sec) } \\
\hline & JM 11.0 & Proposed & JM 11.0 & Proposed & JM 11.0 & Proposed \\
\hline$C$ & 2.52 & 1.92 & 13.24 & 1.0 & 58.87 & 57.69 \\
\hline$F$ & 2.74 & 1.25 & 15.92 & 0.93 & 59.77 & 59.12 \\
\hline$S$ & 1.69 & 0.44 & 10.35 & 0.97 & 60.02 & 58.69 \\
\hline$M$ & 1.49 & 0.45 & 13.12 & 1.33 & 59.7 & 58.19 \\
\hline$G$ & 1.16 & 0.56 & 7.2 & 0.99 & 59.21 & 56.29 \\
\hline
\end{tabular}




\section{Conclusion}

The proposed algorithm enhances the subjective spatial quality by increasingly assigning QP values in xROI's away from ROI, thus making it more visually appealing. Also, it allows for more flexibility in controlling the output bitrates with such QP assignments in ROI and xROI's. The experimental results exhibit that the proposed algorithm is more applicable for video telephony than the original JM 11.0 of H.264|AVC for mobile communication networks with limited transmission bandwidth.

\section{References}

1. ISO/IEC 14496-10, Information Technology-Coding of Audio Visual Objects-part 10: Advanced Video Coding (December 2003)

2. Wiegand, T., Sullivan, G.J., Bjontegard, G., Luthra, A.: Overview of the H.264/AVC Video Coding Standard. IEEE Trans. Circuit System. Video Technology 13, 560-576 (2003)

3. Richardson, I.E.G.: H.264 and MPEG-4 Video Compression, pp. 256-262. Wiley, Chichester (2003)

4. Li, Z.G., Gao, W., Pan, F.: Adaptive rate control for H.264. Visual Communication \& Image Representation, 376-406 (August 2005)

5. He, Z., Kim, Y.K., Mitra, S.K.: Low-Delay Rate Control for DCT Video Coding via $\rho$ domain Source Modeling. IEEE Trans. Circuit System. Video Technology 11, 928-940 (2001)

6. He, Z., Mitra, S.K.: A Unified Rate-Distortion Analysis Framework for Transform coding. IEEE Trans. Circuit System. Video Technology 11, 1221-1236 (2001)

7. He, Z., Chen, T.: Linear rate control for JVT video coding. In: International Conference on Information Technology. Research and Education, Newark (2003)

8. Leuven, S.V., Schevensteen, K.V., Dams Peter, T.: Implementation of Multiple RegionOf-Interest Models in H.264/AVC. In: SITIS, pp. 502-511 (2006)

9. Li, H., Wang, Z., Cui, H., Tang, K.: An Improved ROI-Based Rate Control Algorithm for H.264/AVC. In: IEEE International Conference on Signal Processing 2006 (2006)

10. Liu, Y., Li, Z.G., Soh, Y.C.: Region-of-Interest Based Resource Allocation for Conversational Video Communication of H.264/AVC. IEEE Trans. Circuit System. Video Technology $18,134-139$ (2008)

11. Joint Model - H.264/AVC Reference Software, http://iphome.hhi.de/suehring/download 